\title{
»Dicke Bohnen« als Auslöser einer akuten hämolytischen Anämie
}

\author{
R. L. Riepl, J. Schreiner, B. Müller, S. Hildemann und K. Loeschke \\ Medizinische Klinik (Direktor: Prof. Dr. P. C. Scriba) des Klinikums Innenstadt der Universität München
}

\begin{abstract}
Bei einem bisher gesunden 17jährigen Griechen trat akut eine Gelbverfärbung der Skleren und der Haut auf. Zudem war er auffallend blaß bei sonst unauffälligem körperlichem Untersuchungsbefund. Er berichtete ferner über Müdigkeit und Schwäche. Die klinisch-chemische Untersuchung ergab einen Hämoglobinspiegel von $9,6 \mathrm{~g} / \mathrm{dl}$, eine Lactatdehydrogenase-Aktivität von $335 \mathrm{U} / \mathrm{l}$, eine Gesamtbilirubinkonzentration von $3,2 \mathrm{mg} / \mathrm{dl}$ (direkt $0,7 \mathrm{mg} / \mathrm{dl}$, indirekt $2,5 \mathrm{mg} / \mathrm{dl}$ ) und eine Haptoglobinkonzentration von $48,8 \mathrm{mg} / \mathrm{dl}$. Danach wurde eine hämolytische Anämie vermutet; es stellte sich durch gezieltes Nachfragen heraus, daß der Patient 3 und 2 Tage vor Auftreten des Ikterus zum ersten $\mathrm{Mal}$ in seinem Leben etwa $300 \mathrm{~g}$ »dicke Bohnen « gegessen hatte, die in der Literatur als Sau- oder Pferdebohnen (Vicia faba) bezeichnet werden. Der biochemische Nachweis des Fehlens der Glucose-6-Phosphat-Dehydrogenase-Aktivität in den Erythrozyten sicherte die Diagnose Favismus. Die Lactatdehydrogenase-Aktivität und der Bilirubinspiegel fielen unter rein symptomatischer Therapie innerhalb einer Woche in den Normbereich ab, der Hämoglobinspiegel lag nach 4 Wochen wieder bei $15,7 \mathrm{~g} / \mathrm{dl}$.
\end{abstract}

\section{Acute haemolytic anaemia caused by exposure to broad beans}

A previously healthy 17-year-old Greek boy suddenly developed jaundice of sclerae and skin. In addition, physical examination revealed a pale appearance. He also reported feeling tired and weak. . The haemoglobin level was $9.6 \mathrm{~g} / \mathrm{dl}$, lactate dehydrogenase activity $335 \mathrm{U} / \mathrm{l}$, bilirubin concentration $3.2 \mathrm{mg} / \mathrm{dl}$ (direct bilirubin $0.7 \mathrm{mg} / \mathrm{dl}$, indirect bilirubin $2.5 \mathrm{mg} / \mathrm{dl}$ ), haptoglobin concentration $48.8 \mathrm{mg} / \mathrm{dl}$. As haemolytic anaemia was assumed, direct questioning elecited the fact that the patient had, for the first time in his life, eaten $300 \mathrm{~g}$ of broad beans (Vicia faba) on each of two days, namely 3 and 2 days before the appearance of jaundice. Absence of glucose-6phosphate dehydrogenase activity in the red blood corpuscles confirmed the diagnosis of favism. On symptomatic treatment both the enzyme activities and the bilirubin level fell to normal within one week, and the haemoglobin level was $15.7 \mathrm{~g} / \mathrm{dl}$ after 4 weeks.
Durch die weltweit erhöhte Mobilität müssen sich Ärzte in zunehmendem Maße auch mit Krankheiten auseinandersetzen, die im eigenen Land sehr selten oder gar nicht, im Herkunftsland des Patienten aber häufiger vorkommen. Im folgenden wird anhand eines Fallberichtes eine erbliche hämolytische Anämie vorgestellt, die bei Bewohnern des Mittelmeerraumes nicht selten beobachtet wird.

Dtsch. med. Wschr. 118 (1993), 932-935

(c) Georg Thieme Verlag Stuttgart · New York

\section{Kasuistik \\ Anamnese}

Ein 17jähriger Grieche, der seit 6 Jahren mit seinen Eltern in Deutschland lebt, kam zur Notaufnahme mit einer Gelbverfärbung der Haut und der Skleren, die er am Vortag erstmals bemerkt hatte. Der Urin war dunkler geworden, die Farbe des Stuhls normal geblieben. Er berichtete über allgemeines Krankheitsgefühl mit Müdigkeit und Schwäche. Juckreiz der Haut, Blut-, Leber- oder Gallenwegserkrankungen sowie die Übertragung von Blut oder Blutprodukten verneinte er. 3 Wochen zuvor hatte der junge Mann wegen einer Erkältung mit Rachenschmerzen mehrere Tabletten Acetylsalicyl- 
säure und drei Tabletten Phenoxymethylpenicillin eingenommen. Ein Auslandsaufenthalt war nicht vorausgegangen. Der Patient arbeitet als Küchengehilfe. Er betreibt Bodybuilding, versicherte aber, keine Anabolika einzunehmen. Die in Deutschland lebenden Eltern und die Schwester sowie ein in Griechenland gebliebener Bruder sind gesund und hatten bisher keine Gelbsucht.

\section{Klinischer Aufnahmebefund}

Der $72 \mathrm{~kg}$ schwere, $170 \mathrm{~cm}$ große, kräftige Patient war in gutem Allgemeinzustand. Der Blutdruck betrug $130 / 60 \mathrm{~mm} \mathrm{Hg}$, die Pulsfrequenz $88 / \mathrm{min}$. Auffallend waren ein deutlich sichtbarer Haut- und Sklerenikterus sowie eine Blässe der Haut. Die Auskultation von Herz und Lunge ergab einen regelrechten Befund. Leber und Milz sowie Lymphknoten waren palpatorisch nicht vergrößert. Die übrige internistische und orientierend neurologische Untersuchung erbrachte keinen pathologischen Befund.

Die erste Verdachtsdiagnose lautete auf infektiöse (virale) Hepatitis, differentialdiagnostisch auch Hämolyse mit Anämie.

\section{Klinisch-chemische Untersuchungen}

Im Normbereich lagen die Erythrozytenindices, die Serumspiegel der Elektrolyte, der Retentionswerte, der Transaminasen und Cholestaseparameter, die Gerinnungszeiten, die Serumkonzentrationen des Komplement C3 und C4, des Eisens, Ferritins, Vitamins $B_{12}$, der Folsäure sowie die Hämoglobin-Elektrophorese. Der direkte und indirekte Coombs-Test sowie die Hepatitisserologie und der Suchtest auf antinukleäre Antikörper verliefen negativ

Pathologisch verändert waren die $\mathrm{Hä-}$ moglobinkonzentration $(9,6 \mathrm{~g} / \mathrm{dl}$, Normwert $14-18)$, die Erythrozytenzahl (2,9 T/1; 4,4-6,3 [Retikulozytenzahl $8 \%$ l) sowie die Serumkonzentrationen der Lactatdehydrogenase (335 U/1, 80-240), des Gesamtbilirubins $(3,2 \mathrm{mg} / \mathrm{dl},<1,1)$, des direkten $(0,7 \mathrm{mg} / \mathrm{dl})$ und des indirekten Bilirubins $(2,5 \mathrm{mg} / \mathrm{dl})$ sowie des Haptoglobins $(48,8 \mathrm{mg} / \mathrm{dl}, 60-270)$. Gering erhöht war die osmotische Resistenz der Erythrozyten; der Urin war im Schnelltest zweifach positiv für Bilirubin und einfach positiv für Urobilinogen.

\section{Ergänzende Untersuchungen}

Das Röntgenbild des Thorax ergab eine kleine Verkalkung im rechten Unterfeld. Das Elektrokardiogramm erbrachte einen Normalbefund, die Oberbauchsonographie lediglich eine gering vergröBerte Milz $(12 \times 4,5 \times 8 \mathrm{~cm})$.

\section{Diagnose}

Durch eine gezielte Anamnese wurde herausgefunden, daß der Patient 3 und 2 Tage vor dem Auftreten des Ikterus jeweils etwa $300 \mathrm{~g}$ tiefgefrorene, in Wasser erhitzte "grüne Bohnen « gegessen hatte. Auf einer angeforderten Verpackung war die Aufschrift »Dicke Bohnen « zu lesen, bei denen es sich um Saubohnen handelte. Die Bestimmung der Glucose-6-Phosphat-Dehydrogenaseaktivität (G-6PD) in den Erythrozyten ergab ein vollständiges Fehlen dieser Enzymaktivität und sicherte die Diagnose einer akuten hämolytischen Anämie durch Favismus.

\section{Therapie und Verlauf}

Die Therapie der akuten Hämolyse bei Glucose-6-Phosphat-Dehydrogenase-Mangel ist rein symptomatisch. Die Verabreichung von Erythrozytenkonzentraten war nicht erforderlich. Die Hämoglobinkonzentration stieg vom tiefsten Wert $(8,9 \mathrm{~g} / \mathrm{dl}$, $89 \%$ Retikulozyten) wieder kontinuierlich an und lag bei einer ambulanten Kontrolluntersuchung 4 Wochen später wieder im Normbereich $(15,7 \mathrm{~g} / \mathrm{dl}$, $7 \%$ o Retikulozyten, entsprechend früheren hausärztlichen Kontrollen). Auch die LactatdehydrogenaseAktivität sowie der Bilirubinspiegel und der Urinbefund normalisierten sich innerhalb einer Woche, und der Patient konnte beschwerdefrei in gutem Allgemeinzustand entlassen werden. Es wurde ihm empfohlen, »dicke Bohnen « (Saubohnen) und bestimmte Medikamente wie Primaquin, Nalidixinsäure, Nitrofurantoin in Zukunft zu meiden.

\section{Diskussion}

Die erste Verdachtsdiagnose einer viralen Hepatitis erwies sich aufgrund normaler Aktivitäten der Leberenzyme und einer negativen Hepatitisserologie rasch als falsch. Die klinisch-chemischen Befunde belegten eine akute Hämolyse; als deren Ursache lag bei dem ansonsten gesunden jungen Mann zunächst eine Nebenwirkung der eingenommenen Medikamente nahe. Da jedoch ein langer zeitlicher Abstand zwischen der Einnahme von Acetylsalicylsäure und Penicillin und dem Beginn der Symptome lag, erschien dies doch eher unwahrscheinlich. Zudem versicherte der Patient glaubhaft, keine anderen Medikamente eingenommen zu haben. Eine immunologische Ursache (Autoimmunhämolyse) oder eine Anomalie der Erythrozytenform (beispielsweise Sphärozytose) waren klinisch-chemisch und klinisch nahezu ausgeschlossen. Die griechische Herkunft des Patienten lenkte den Verdacht trotz leerer Familienanamnese schließlich auf hämolytische Krankheiten, die in den Mittelmeerländern häufiger vorkommen (beispielsweise Favismus, Thalassämie). Eine Thalassämie konnte durch eine normale Erythrozytenform und unauffällige Hämoglobinelektrophorese ausgeschlossen werden, so daß 
angesichts des zeitgleichen Genusses von Bohnen ein Favismus recht wahrscheinlich erschien. Schwierigkeiten bereitete die definitive Identifikation der verzehrten »dicken Bohnen« als Saubohnen. Die Abbildung auf der Verpackung zeigte lediglich grüne, etwa $1 \mathrm{~cm}$ große Bohnenkerne mit einer Einkerbung um den halben Durchmesser. Nachforschungen in der Literatur für die Vollwertküche (3) ergaben, daß die Bezeichnung »dicke Bohnen« wohl aus Gründen der Verbraucherfreundlichkeit gleichbedeutend mit Sauoder Pferdebohnen Verwendung findet. Saubohnen haben eine grüne Farbe, wenn sie jung und frisch sind, getrocknet sind sie bräunlich. Die wissenschaftlich botanische Bezeichnung der Saubohne ist Vicia faba (6). Ein Favismus wurde im geschilderten Fall nachgewiesen durch das vollständige Fehlen der Glucose-6-Phosphat-Dehydrogenase-Aktivität in den Erythrozyten.

Pathophysiologisch liegt dem Favismus ein Mangel oder ein Fehlen der Glucose-6-Phosphat-Dehydrogenase-Aktivität in den Erythrozyten zugrunde. Der Enzymdefekt wurde durch Beutler (1) in den fünfziger Jahren erstmals beschrieben. Die hämolytische Wirkung des Antimalariamittels Primaquin bei schwarzen US-Soldaten im Koreakrieg konnte er auf diesen Enzymdefekt zurückführen. Der Favismus wird $X$-chromosomal rezessiv vererbt und führt beim männlichen Geschlecht zu Krankheitszeichen. Heterozygote Konduktorinnen weisen ein stark wechselndes Mosaik mit normalen und enzymdefekten Erythozyten auf, erkranken aber nicht. Unter der weißen Bevölkerung schwankt die Prävalenz zwischen $<0,1 \%$ bei Nordeuropäern und $50 \%$ bei kurdischen Juden. Unter den Mittelmeerbewohnern liegt sie zwischen $1 \%$ (Spanier) und $18 \%$ (Griechen), bei schwarzen Amerikanern um 11\% (5). Beschrieben wurden über 350 biochemisch definierte Varianten der Glucose-6-Phosphat-Dehydrogenase (2). Bei Gesunden wird normalerweise die G-6-PD B beobachtet; die »G-6-PD Mittelmeer« weist eine gegen Null gehende Enzymaktivität (wie im beschriebenen Fall) bei normaler Enzymmenge auf. Bei schwarzen Amerikanern wiederum ist die häufig vorkommende G-6-PD A, die eine normale Aktivität hat, mengenmäßig in den Erythrozyten aufgrund einer In-vivoInstabilität um 85-95\% reduziert. Ein akutes Hämolyse-Syndrom wegen unzureichender Glucose-6Phosphat-Dehydrogenase-Aktivität kann von der Kindheit bis ins Erwachsenenalter, abhängig von der auslösenden Noxe, eintreten. Typische Auslöser sind:

- Medikamente (beispielsweise Nalidixinsäure, Nitrofurantoin, Niridazol, Primaquin, Sulfamethizol, Sulfasalazin und fraglich Acetylsalicylsäure): Hämolysebeginn nach 1 bis 3 Tagen mit Bauchund Rückenschmerzen, Dunkelverfärbung des Urins, starkem Abfall der Hämoglobinkonzentration und Auftreten von Heinz-Innenkörpern;

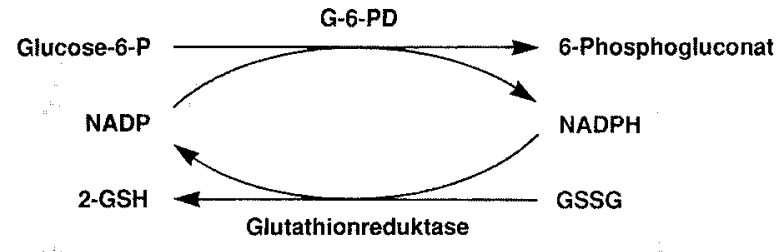

Abb. 1 Schematische Darstellung des Stoffwechsels, durch den Reduktionsäquivalente in Form von NADPH und Glutathion (GSH) in den Erythrozyten gebildet werden. NADPH und GSH wirken den durch Oxidation hervorgerufenen Schäden am Hämoglobin und an der Zellmembran entgegen (G-6-PD = Glucose-6-Phosphat-Dehydrogenase; GSSG = Glutathiondisulfid)

- Favabohne (Saubohne oder dicke Bohne): Hämolysebeginn nach 1 bis 2 Tagen, manchmal auch heftig innerhalb von Stunden mit Schockgefahr, Dunkelverfärbung des Urins, Erstmanifestation meist in der Kindheit;

- Infektionen (beispielsweise Pneumonie, Typhus): Hämolysebeginn einige Tage nach der fieberhaften Erkrankung mit mildem Abfall der Hämoglobinkonzentration und geringem Ikterus;

- Neugeborenenperiode: milde bis keine Anämie mit starkem, verlängertem Ikterus, bei dem die Gefahr eines Kernikterus droht.

Der genaue Hämolysemechanismus ist unbekannt. Die Glucose-6-Phosphat-Dehydrogenase, das Schlüsselenzym des oxidativen Pentose-Phosphat-Zyklus, oxidiert Glucose-6-Phosphat zu 6-Phosphogluconat und erzeugt dabei ein Reduktionsäquivalent in Form von NADPH. In einem weiteren Schritt entsteht dann nochmals ein Äquivalent NADPH. Diese Reduktionsäquivalente dienen in den Erythrozyten als Co-Enzyme der Methämoglobinreduktase, der Diaphorase und der Glutathionreduktase, die für die ständige Beseitigung von Oxidationsschäden durch Sauerstoff am Hämoglobin und an der Zellmembran sorgen (Abbildung 1). Die Einnahme bestimmter Medikamente führt zur vermehrten Bildung stark oxidativen Wasserstoffperoxids, das durch Glutathion nicht mehr vollständig reduziert werden kann, da Glutathion seinerseits mangels NADPH nur noch ungenügend aus Glutathiondisulfid $(=$ oxidiertes Glutathion) regeneriert wird. Man nimmt an, daß die dadurch entstehenden oxidativen Membranschäden zum akuten Untergang bevorzugt älterer (schon geschädigter) Erythrozyten führen. Bei der infektionsinduzierten Hämolyse kann vermutlich ebenfalls Wasserstoffperoxid, das durch phagozytierende Leukozyten entsteht, nur unvollständig reduziert werden. Etwas anders scheint der Hämolysemechanismus beim Favismus zu sein. Die Saubohne enthält nämlich eine Substanz, die reduziertes Glutathion inaktiviert (4), welches mangels NADPH nicht mehr regeneriert werden kann. 


\section{Literatur}

1 Beutler, E.: The hemolytic effect of primaquine and related compounds. A review. Blood 14 (1959), 103-139.

2 Beutler, E.: Glucose-6-phosphate dehydrogenase deficiency. In Williams, W. J., E. Beutler, A. J. Ersley, M. A. Lichtman (Ed.): Hematology (McGraw-Hill: New York 1990), 591-606.

3 v. Eichborn, B.: Erbsen, Linsen, Bohnen in der Vollwertküche (Gräfe \& Unzer: München 1988).

4 Harley, J. D., A. M. Mauer: Studies in the formation of Heinz bodies. I. Methemoglobin production and oxyhemoglobin destruction. Blood 16 (1960), 1722-1735.

5 Luzzatto, L., G. Battistuzzi: Glucose-6-phosphate dehydrogenase. In Harris, H., K. Hirschhorn (Ed.): Advances in Human Genetics (Plenum: New York 1985), 217-329.

6 Schmeil, J., J. Fitschen: Flora von Deutschland und seinen angrenzenden Gebieten (Meyer: Heidelberg 1968).
Dr. R. L. Riepl, Dr. Josefine Schreiner, Beate Müller, S. Hildemann, Prof. Dr. K. Loeschke

Medizinische Klinik

Klinikum Innenstadt der Universität

Ziemssenstr. 1

W-8000 München 2 